\title{
Descripción de los resultados y costes de una intervención preventiva a nivel respiratorio en el anciano institucionalizado: ensayo controlado aleatorizado
}

\author{
Maria dels Àngels Cebrià i Iranzo a,b,*, M. Ángeles Tortosa-Chuliác, Celedonia Igual-Camacho ${ }^{\mathrm{a}, \mathrm{d}}$, \\ Patricia Sancho ${ }^{\mathrm{e}}$, Laura Galiana ${ }^{\mathrm{e}}$ y José Manuel Tomás ${ }^{\mathrm{e}}$ \\ a Departament de Fisioteràpia, Universitat de València, Valencia, España \\ b Servicio de Rehabilitación, Hospital Universitari i Politècnic La Fe, Valencia, España \\ ${ }^{\mathrm{c}}$ Departament d'Economia Aplicada, Universitat de València, Valencia, España \\ d Servicio de Rehabilitación, Hospital Clínic Universitari, Valencia, España \\ e Departament de Metodologia de les Ciències del Comportament, Universitat de València, Valencia, España
}

\section{INFORMACIÓN DEL ARTÍCULO}

\section{Historia del artículo:}

Recibido el 30 de agosto de 2013

Aceptado el 19 de noviembre de 2013

On-line el $\mathrm{xxx}$

\section{Palabras clave:}

Análisis coste-consecuencia

Función de la musculatura respiratoria

Fisioterapia

Anciano

Residencias

\begin{abstract}
R E S U M E N
Introducción: En el anciano institucionalizado con limitación funcional se evidencia una mayor reducción de la funcionalidad de la musculatura respiratoria (MR). Los objetivos de este estudio son evaluar los resultados y costes de una intervención de entrenamiento de la MR mediante Pranayama en población anciana institucionalizada con limitación funcional.

Material y métodos: Estudio controlado aleatorizado desarrollado en ancianos institucionalizados con limitación para la deambulación $(n=54)$. La intervención consistió en el entrenamiento de la MR mediante Pranayama, durante 6 semanas ( 5 sesiones/semana). Los resultados se midieron en relación con la función de la MR mediante las presiones inspiratoria y espiratoria máximas y la ventilación máxima voluntaria, en 4 tiempos. También se valoró la satisfacción percibida por el grupo experimental (GE) a través de un cuestionario ad hoc. Se estimaron los costes directos e indirectos de la intervención desde la perspectiva social.

Resultados: El GE reveló una mejora significativa de la fuerza (presiones inspiratoria y espiratoria máximas) y de la resistencia (ventilación máxima voluntaria) de la MR. Además, un 92\% del GE refirió una satisfacción alta. Los costes sociales totales, directos e indirectos, ascendieron a $21.678 €$.

Conclusiones: Esta evaluación revela que los resultados en términos de la función de la MR son significativos, que la intervención es bien tolerada y valorada por el residente, y los costes de la intervención son moderados.
\end{abstract}

(c) 2013 SEGG. Publicado por Elsevier España, S.L. Todos los derechos reservados.

\section{Cost-consequence analysis of respiratory preventive intervention among institutionalized older people: Randomized controlled trial}

\section{A B S T R A C T}

Introduction: The institutionalized elderly with functional impairment show a greater decline in respiratory muscle (RM) function. The aims of the study are to evaluate outcomes and costs of RM training using Pranayama in institutionalized elderly people with functional impairment.

Material and methods: A randomized controlled trial was conducted on institutionalized elderly people with walking limitation $(n=54)$. The intervention consisted of 6 weeks of Pranayama RM training ( 5 times/week). The outcomes were measured at 4 time points, and were related to RM function: the maximum respiratory pressures and the maximum voluntary ventilation. Perceived satisfaction in the experimental group (EG) was assessed by means of an ad hoc questionnaire. Direct and indirect costs were estimated from the social perspective.
Cost-consequence analysis

Respiratory muscle function

Physiotherapy

Nursing homes
Nurder people 
Results: The GE showed a significant improvement related with strength (maximum respiratory pressures) and endurance (maximum voluntary ventilation) of RM. Moreover, 92\% of the EG reported a high satisfaction. The total social costs, direct and indirect, amounted to $€ 21,678$.

Conclusions: This evaluation reveals that RM function improvement is significant, that intervention is well tolerated and appreciated by patients, and the intervention costs are moderate.

(C) 2013 SEGG. Published by Elsevier España, S.L. All rights reserved.

\section{Introducción}

En el anciano institucionalizado el deterioro de las funciones motoras repercute de manera relevante en el desempeño de las actividades de la vida diaria $(A V D)^{1}$. A este respecto, algunos estudios $^{2,3}$ señalan que la función de la musculatura respiratoria (MR) es significativamente mayor en el anciano físicamente activo que en el sedentario, y asocian la limitación para deambular y otras AVD con reducción de la funcionalidad de la MR. En esta línea, Simões et al. ${ }^{4}$ evidencian la prevalencia de la reducción de la fuerza de la MR en las mujeres entre 60 y 89 años institucionalizadas, y enfatizan la necesidad de mejorar la función de la MR en esta población, para prevenir las complicaciones directamente relacionadas con esta reducción, por ejemplo la insuficiencia respiratoria. La mejora en la función respiratoria a través del entrenamiento de la MR ha sido estudiada fundamentalmente en el anciano sin limitación funcional y que vive en comunidad ${ }^{5-7}$. Un estudio previo analiza los resultados de esta intervención respiratoria en el anciano institucionalizado con limitación para deambular ${ }^{8}$. Existen diversas técnicas respiratorias, algunas ampliamente utilizadas como el dispositivo de carga umbral ${ }^{9,10}$, y otras menos conocidas como realizar ejercicios de control respiratorio (en yoga se denomina Pranayama), que pueden ser una alternativa útil para mejorar la MR en la población especialmente debilitada y/o con importante limitación para las AVD. Los resultados asociados con esta última técnica (a nivel respiratorio: descenso de la frecuencia respiratoria y mejora de la función muscular - presiones respiratorias máximas y ventilación máxima voluntaria) son corroborados por estudios previos en población diversa ${ }^{11-14}$, pero no en el anciano institucionalizado con limitación funcional. En consecuencia, este estudio se centra en evaluar una intervención respiratoria mediante Pranayama en esta población concreta.

Por otra parte, este estudio pretende colaborar en la mejora de toma de decisiones en las residencias. En España se está introduciendo un nuevo modelo de atención más cercano a las preferencias de las personas mayores. Este modelo denominado "Atención gerontológica centrada en la persona» ${ }^{15}$ promueve cambios en la gestión residencial. Uno de ellos consiste en seguir de cerca el desarrollo de las actividades del centro y evaluar los resultados y costes derivados de las mismas. La Asociación de Economía de la Salud ${ }^{16}$ recomienda la evaluación económica de las intervenciones en la gestión de servicios sanitarios y sociales para tomar decisiones privadas o públicas más sensatas. En este sentido, la Fundación Matia ${ }^{17}$ ha realizado una revisión de la literatura a nivel internacional sobre intervenciones implementadas recientemente centradas en la persona. Y de ella se extrae la necesidad de realizar evaluación económica en el ámbito residencial y en las actividades de rehabilitación, porque son bastante escasas. Además, la $\mathrm{OCDE}^{18}$ recomienda que se exija información sobre una serie de indicadores de calidad a las residencias y demás servicios sociosanitarios para personas mayores. Existen múltiples técnicas de evaluación económica a utilizar por parte de los gestores. El presente estudio ha escogido una técnica sencilla, que no aplica criterios de selección como la efectividad o la eficiencia, y clasificada como evaluación económica parcial según Drummond et al. ${ }^{19}$. Consiste en la descripción de los costes y de los resultados de una intervención. Esta técnica, también denominada análisis coste-consecuencia, busca facilitar a los responsables la interpretación de las conclusiones de las evaluaciones económicas (sobre todo ante resultados que no necesitan traducirse en valores monetarios y son complicados de obtener en determinados ámbitos sanitarios y grupos de población $)^{20,21}$. El análisis coste-consecuencia ha sido aplicado previamente en el ámbito de rehabilitación y de los servicios sociosanitarios destinados a los mayores ${ }^{22-24}$.

Los objetivos de este estudio son: 1) evaluar los resultados de una intervención de entrenamiento de la MR mediante Pranayama en población anciana institucionalizada con limitación funcional; y 2) describir y estimar los costes de esta actividad preventiva respiratoria.

\section{Material y métodos}

Diseño

El presente estudio es un estudio controlado aleatorizado, aprobado por el Comité Ético de Investigación en Humanos de la Universitat de València, y desarrollado durante el primer semestre de 2009. Todos los procedimientos de reclutamiento, medición y entrenamiento aplicados han respetado las normas éticas de la Declaración de Helsinki.

\section{Participantes}

La muestra reclutada es de 54 ancianos institucionalizados procedentes de 2 residencias de Valencia (España). Ambos centros seguían criterios similares de admisión-clasificación de residentes, servicios de atención sociosanitaria y cualificación de los profesionales. Los centros pertenecían al ámbito privado y sector solidario, con plazas concertadas. Según el número de plazas de que disponen, eran calificados como centros de tamaño mediano (entre $50-70$ plazas), y alrededor de un $60 \%$ de las plazas eran para usuarios con alta dependencia, grados II (severa) y III ${ }^{25}$. El reclutamiento se realizó atendiendo a los siguientes criterios de inclusión: a) incapacidad para deambular más de $10 \mathrm{~m}$, o el uso de silla de ruedas; b) puntuación del Mini-Examen Cognoscitivo de Lobo $^{26} \geq 20$ puntos, para asegurar la adecuada colaboración en las pruebas de medición y entrenamiento. A continuación, se completó un cuestionario sobre el historial médico de los residentes para identificar condiciones de inestabilidad clínica que pudieran excluirles: a) problemas cardiorrespiratorios crónicos (p. ej., bloqueos auriculoventriculares y fibrilación auricular); b) episodio cardiorrespiratorio agudo (2 meses previos); c) problemas neurológicos, musculares o neuromusculares que pudieran interferir en las pruebas de medición o entrenamiento; d) fumadores activos o exfumadores ( $\leq 5$ últimos años); y e) enfermos terminales. De los residentes potenciales, tan solo aquellos que cumplieron los criterios de inclusión y no presentaron ninguno de los criterios de exclusión fueron finalmente reclutados. Esto es, la muestra que participó en el estudio fue alrededor del 50\% del número de residentes total.

Los residentes fueron asignados, a través del generador de números aleatorios del programa informático SPSS, a 2 grupos: control (GC, $n=27)$ y experimental $(G E, n=27)$. Para el cálculo del tamaño muestral, y dado que no existían estudios de condiciones 
similares a este, se acudió a literatura previa ${ }^{11-13}$ que mostraba efectos significativos de la técnica. Se optó por un tamaño muestral superior, que tuviera mayor potencia estadística que la de estos estudios para el mismo tamaño del efecto.

\section{Programa de prevención con entrenamiento de la musculatura respiratoria}

La intervención consistió en el entrenamiento supervisado de la MR mediante ejercicios de respiración controlada (Pranayama). El protocolo se aplicó tan solo en el GE, 5 sesiones matutinas por semana, de 30 min de duración, durante 6 semanas consecutivas. El programa de ejercicios respiratorios fue diseñado por un profesor de yoga cualificado (Yoga Siromani) y desarrollado por fisioterapeutas formados. El programa se centró en la respiración nasal lenta y profunda, realizando una espiración activa en todos los ejercicios, y sin incluir Yogasanas (práctica de diferentes posturas del cuerpo en bipedestación, sedestación y decúbito) ni meditación, a diferencia de estudios previos ${ }^{11,13}$. La dificultad de los ejercicios se aumentó semanalmente en función de los parámetros: a) aumento en el número de repeticiones (volumen de trabajo); b) aumento de la resistencia al respirar, pasando de utilizar 2 a una narina; c) intercalando tiempos de apnea y aumentando los tiempos del ciclo ventilatorio (inspiración: apnea postinspiración: espiración: apnea postespiración). El fisioterapeuta que supervisaba cada sesión recogió diariamente datos de la percepción subjetiva de esfuerzo según la escala Borg-CR10 ${ }^{27}$. Una vez por semana, se obtuvo un registro de la saturación de oxígeno y la frecuencia cardíaca, antes e inmediatamente después de cada sesión de entrenamiento mediante el pulsioxímetro (SmartOx WM18000, Weinmann Medical Technology ${ }^{\circledR}$, Alemania), con objeto de garantizar la seguridad de la intervención.

\section{Mediciones}

Esta evaluación consistió solo en describir y estimar los resultados y costes derivados de esta intervención. No es una evaluación económica completa como el análisis coste-efectividad, pues no busca comparar los ratios incrementales de costes/resultados de diferentes intervenciones ${ }^{19}$.

Todos los participantes fueron medidos en 4 tiempos: preentrenamiento (tiempo 0), intermedio (semana 4), postentrenamiento (semana 7) y seguimiento (semana 10). Las variables resultado principales fueron: presión inspiratoria máxima (PImáx), presión espiratoria máxima (PEmáx) y ventilación máxima voluntaria (MVV). Como resultado secundario se valoró la calidad de la intervención recibida por el GE, a través de preguntas ad hoc, y del nivel de esfuerzo que realizaron los mayores durante las sesiones. Por otra parte, se estimaron los costes directos e indirectos de la intervención desde la perspectiva social (costes de los profesionales sanitarios, residentes y familias). En el estudio colaboraron 2 fisioterapeutas especializados en el ámbito respiratorio para realizar el reclutamiento y las mediciones, y 2 fisioterapeutas especializados en geriatría para supervisar el entrenamiento (no miembros de la plantilla de las residencias participantes). Los profesionales que realizaron las mediciones no participaron en el entrenamiento.

La función pulmonar se valoró mediante un espirómetro portátil Jaeger (VIASYS ${ }^{\circledR}$ Healthcare GmbBH, Hoechberg, Alemania). La PImáx y la PEmáx se midieron a través de un manómetro aneroide (Series 2000 Magnehelic ${ }^{\circledR}$ Pressure Gauge, Dwyer Instruments, Michigan City, Indiana, EE. UU.) con rango de sensibilidad de entre 0 a $300 \mathrm{~cm} \mathrm{H}_{2} \mathrm{O}$ de presión. Los valores de referencia utilizados para obtener los porcentajes fueron los recogidos por Enright et al. ${ }^{28}$. La MVV, volumen máximo de aire que una persona ventila voluntariamente durante un intervalo de $12 \mathrm{~s}$, se valoró mediante el espirómetro portátil Jaeger anteriormente especificado. Los valores de referencia empleados para obtener los porcentajes fueron los publicados por Neder et al. ${ }^{29}$. Todas las mediciones, la espirometría, así como la PImáx, la PEmáx y la MVV, siguieron los estándares requeridos por la American Thoracic Society y la European Respiratory Society $^{30,31}$

La calidad del servicio fue medida a través del grado de satisfacción percibido por los residentes del GE y se obtuvo a partir de la contestación a 2 preguntas al finalizar el programa de entrenamiento (semana 7): 1) Si tuviera que decidir ahora participar en este protocolo de ejercicios respiratorios, ¿cuál sería su contestación?; y 2) ¿Cómo de satisfecho está de haber participado en el protocolo de ejercicios respiratorios?, 1 es "Nada satisfecho» y 10 es "Muy satisfecho".

Los costes directos de los profesionales fueron aquellos relacionados con su retribución, material/equipamiento, formación, organización y transporte, y se estimaron en euros del año 2009, según los siguientes parámetros: a) salario base del fisioterapeuta en residencias ${ }^{32}(1.108,44 € /$ mes, o $40 \mathrm{~h} / \mathrm{mes} \Rightarrow 27,7 € / \mathrm{h})$, utilizado para el cálculo de los costes de organización y retribución; b) precio medio de curso estándar de formación en Pranayama, 5 sesiones individuales de $2 \mathrm{~h}(200 €)$; c) bonometro Valencia ${ }^{33}$ (bonometro 10 viajes, zona-AB 9,30€); d) tasa de kilometraje para desplazamientos en coche particular ${ }^{34}(0,19 € / \mathrm{km})$; e) facturas de materiales/equipos necesarios para las mediciones y el entrenamiento (espirómetro, manómetro anaeroide, boquillas-filtro y pulsioxímetro). Los costes indirectos de estos fisioterapeutas fueron sus costes de oportunidad, es decir el valor del trabajo que estarían efectuando en otro lugar distinto al que realizan en estas residencias (estimado según salario base del fisioterapeuta convenio colectivo sanidad privada en $2009^{35}: 1.210,24 € /$ mes, o $40 \mathrm{~h} / \mathrm{mes} \Rightarrow 30,25 € / \mathrm{h})$. En lo que respecta a los residentes, los costes directos se relacionaron con el esfuerzo percibido durante el entrenamiento (según la escala Borg-CR10 27 ). Sus costes indirectos fueron aquellos derivados de lo que dejarían de hacer en la residencia cuando acuden a la sesión de entrenamiento (normalmente participar en otras actividades lúdicas). Este coste se estimó según el salario base del trabajador social en residencias ${ }^{32}$ $(1.108,44 € /$ mes, o $40 \mathrm{~h} / \mathrm{mes} \Rightarrow 27,7 € / \mathrm{h})$. Finalmente, los costes directos e indirectos relacionados con la familia fueron los asociados a su participación en actividades cotidianas y sociales con sus parientes en la residencia. Al tratarse de una intervención de duración menor de un año, no hay necesidad de aplicar tipos de descuento a los costes.

\section{Análisis estadístico}

Los análisis estadísticos se realizaron mediante el programa SPSS versión 20. Se calcularon los estadísticos descriptivos de todas las variables (medias, desviaciones típicas, porcentajes e intervalos de confianza del 95\% [IC95\%]). Se emplearon las técnicas estadísticas y gráficas recomendadas para evaluar supuestos de normalidad, homocedasticidad, etc. y valores atípicos. Adicionalmente, se emplearon técnicas inferenciales (pruebas $t$ y chi-cuadrado) para comparar ambos grupos en la línea base, así como análisis de varianza (ANOVA) mixtos 2 (grupo) $\times 4$ (tiempo) para analizar los efectos de la intervención mediante Pranayama vs. control. Se calcularon pruebas post-hoc ante diferencias estadísticamente significativas con corrección de Bonferroni, así como estimaciones del tamaño del efecto mediante eta al cuadrado parcial $\left(\eta^{2}\right)$. En el caso de las variables que no siguieron una distribución normal, se utilizaron pruebas no paramétricas (Mann-Whitney para comparar los grupos en variables cuantitativas). 


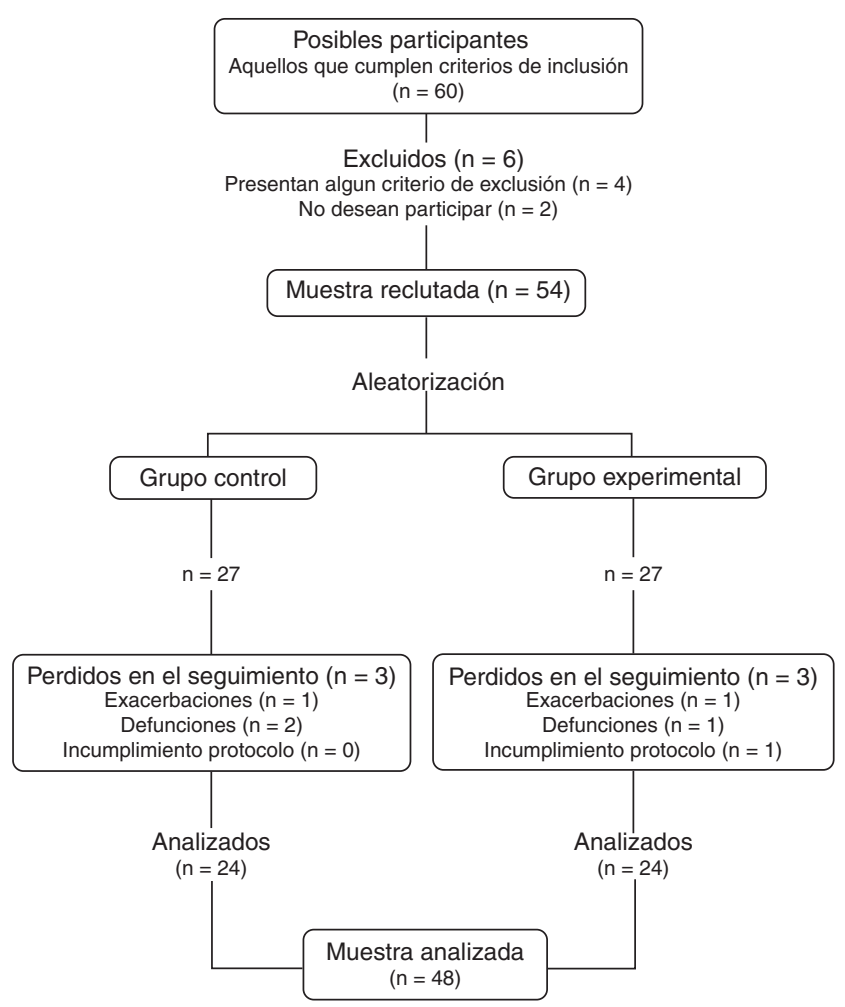

Figura 1. Diagrama de flujo de los participantes.

\section{Resultados}

La muestra reclutada fue de 54 ancianos institucionalizados, de $85 \pm 6$ años de edad media, un 93\% mujeres. Un total de 48 residentes completaron el estudio (fig. 1). Las características basales demográficas y clínicas se muestran en la tabla 1. El 70\% de la muestra analizada utilizaba silla de ruedas de forma permanente, debido a secuelas de accidente cerebrovascular (47\%), úlcera-amputación de la extremidad inferior por diabetes mellitus (13\%) y enfermedades osteoarticulares (10\%). Respecto a la función pulmonar, la capacidad vital máxima y forzada fue ligeramente mayor en el GE, pero solo para los valores absolutos. En la función de la MR se observó una reducción generalizada del 30\% para la PImáx, un 40\% para la PEmáx y un 65\% para la MVV.

En lo que respecta a la capacidad funcional y cognitiva, no se observaron diferencias significativas entre los grupos: índice de Barthel (GC=73,8 $\pm 18,5$ y GE $=75,0 \pm 20,0$; $p=0,823)$ y MiniExamen Cognoscitivo de Lobo $(\mathrm{GC}=23,8 \pm 3,8$ y $\mathrm{GE}=26,0 \pm 2,4$; $\mathrm{p}=0,078$ )

\section{Efectos de la intervención}

En cuanto a la variable relativa a la fuerza de la musculatura inspiratoria (PImáx, $\mathrm{cm} \mathrm{H}_{2} \mathrm{O}$ ), el GE mostró un incremento progresivo a lo largo del programa de entrenamiento y se observó una tendencia a la estabilización durante el periodo de seguimiento (fig. 2). Este comportamiento de mejora de la PImáx ( $\left.\mathrm{cm} \mathrm{H}_{2} \mathrm{O}\right)$ se traduce en diferencias significativas entre los grupos para las mediciones postentrenamiento (GC=37,3 $\pm 20,2$ [IC95\%: 29,4-45,2] y $\mathrm{GE}=50,4 \pm 18,1$ [IC95\%: 42,5-58,3]; $\mathrm{p}=0,022$ ) y de seguimiento (GC $=34,8 \pm 22,0$ [IC95\%: 26,4-43,2] y GE=51,3 $\pm 18,5$ [IC95\%: $42,9-56,6] ; p=0,007)$. Este resultado indica que el cambio a lo largo del tiempo en el GE es mayor y más estable, puesto que solo el GC vuelve a bajar en el seguimiento. El ANOVA estimado para evaluar el efecto de interacción, el que muestra si el tratamiento resulta
Tabla 1

Características basales de los participantes de ambos grupos estudiados: porcentajes o medias \pm 1 desviación típica

\begin{tabular}{|c|c|c|c|}
\hline Variable & $\begin{array}{l}\text { Grupo } \\
\text { control } \\
(n=24)\end{array}$ & $\begin{array}{l}\text { Grupo } \\
\text { experimental } \\
(n=24)\end{array}$ & Valor $\mathrm{p}$ \\
\hline \multicolumn{4}{|l|}{ Antropométricas } \\
\hline Edad (años) & $86 \pm 5$ & $84 \pm 7$ & 0,286 \\
\hline Altura $(\mathrm{cm})$ & $149,1 \pm 7,1$ & $151,4 \pm 4,9$ & 0,200 \\
\hline Peso (kg) & $65,0 \pm 11,7$ & $65,2 \pm 12,8$ & 0,953 \\
\hline Índice de masa corporal $\left(\mathrm{kg} / \mathrm{m}^{2}\right)$ & $29,2 \pm 4,8$ & $28,5 \pm 4,7$ & 0,567 \\
\hline \multicolumn{4}{|l|}{ Enfermedades diagnosticadas (\%) } \\
\hline Respiratorias & 29 & 25 & 0,745 \\
\hline Cardiovasculares & 79 & 63 & 0,204 \\
\hline Endocrinas & 33 & 33 & 1,000 \\
\hline Neurológicas & 29 & 21 & 0,505 \\
\hline Musculo-esqueléticas & 79 & 54 & 0,066 \\
\hline Otras & 71 & 88 & 0,155 \\
\hline Comorbilidad (número) & $3 \pm 1$ & $3 \pm 1$ & $0,177^{a}$ \\
\hline \multicolumn{4}{|l|}{ Capacidad para deambular (\%) } \\
\hline Bastón & 9 & 13 & 0,671 \\
\hline Andador & 24 & 16 & 0,393 \\
\hline Silla de ruedas & 67 & 71 & 0,452 \\
\hline \multicolumn{4}{|l|}{ Función pulmonar } \\
\hline VC (L) & $1,6 \pm 0,5$ & $1,9 \pm 0,4$ & $0,048^{*}$ \\
\hline VC (\% pred) & $78 \pm 18$ & $91 \pm 22$ & 0,062 \\
\hline FVC (L) & $1,5 \pm 0,4$ & $1,8 \pm 0,4$ & $0,026^{*}$ \\
\hline FVC (\% pred) & $73 \pm 19$ & $86 \pm 21$ & 0,052 \\
\hline $\mathrm{FEV}_{1}(\mathrm{~L})$ & $1,2 \pm 0,4$ & $1,4 \pm 0,4$ & 0,048 \\
\hline $\mathrm{FEV}_{1}$ (\% pred) & $81 \pm 25$ & $94 \pm 26$ & 0,113 \\
\hline $\mathrm{FEV}_{1} / \mathrm{FVC}(\%)$ & $80 \pm 10$ & $80 \pm 9$ & 0,972 \\
\hline $\operatorname{PEF}(\mathrm{L} / \mathrm{s})$ & $2,8 \pm 1,1$ & $3,4 \pm 1,2$ & 0,067 \\
\hline $\operatorname{PIF}(\mathrm{L} / \mathrm{s})$ & $2,0 \pm 0,9$ & $2,2 \pm 0,5$ & 0,271 \\
\hline \multicolumn{4}{|l|}{ Función de la musculatura respiratoria } \\
\hline PImáx ( $\left(\mathrm{cm} \mathrm{H}_{2} \mathrm{O}\right)$ & $30,8 \pm 19,7$ & $33,3 \pm 14,2$ & 0,616 \\
\hline PImáx (\% pred) ${ }^{b}$ & $68 \pm 33$ & $75 \pm 32$ & 0,400 \\
\hline PEmáx $\left(\mathrm{cm} \mathrm{H}_{2} \mathrm{O}\right)$ & $52,1 \pm 24,0$ & $64,4 \pm 26,2$ & 0,096 \\
\hline PEmáx (\% pred $)^{b}$ & $54 \pm 21$ & $67 \pm 28$ & 0,058 \\
\hline $\operatorname{MVV}(\mathrm{L} / \mathrm{min})$ & $30,4 \pm 14,4$ & $33,1 \pm 12,4$ & 0,490 \\
\hline $\operatorname{MVV}(\% \text { pred })^{c}$ & $41 \pm 14$ & $33 \pm 7$ & 0,264 \\
\hline
\end{tabular}

$\mathrm{FEV}_{1}$ : volumen espirado forzado en el primer segundo; FVC: capacidad vital forzada; MVV: ventilación máxima voluntaria; PEF: flujo espiratorio punta; PEmáx: presión espiratoria máxima; PIF: flujo inspiratorio punta; PImáx: presión inspiratoria máxima; VC: capacidad vital; \% pred: porcentajes con respecto a los valores de referencia.

El apartado "Enfermedades diagnosticadas (ED)» muestra los porcentajes de presencia de ED y la media del número de ED (comorbilidad):

a Adicionalmente, se empleó una prueba no paramétrica de Mann-Whitney para comparar la comorbilidad entre los grupos.

b Porcentaje según los valores de referencia de Enright et al. ${ }^{28}$.

c Porcentaje según los valores de referencia de Neder et al. ${ }^{29}$; debe considerarse que solamente se analizó para 10 residentes de los 48 totales (20,8\%) (es decir, solo para aquellos ancianos $<80$ años), pudiendo darse un gran sesgo. Se emplearon la prueba $t$ (variables cuantitativas) y prueba de Chi-cuadrado (variables cualitativas) para obtener las diferencias entre ambos grupos.

${ }^{*} \mathrm{p} \leq 0,05$.

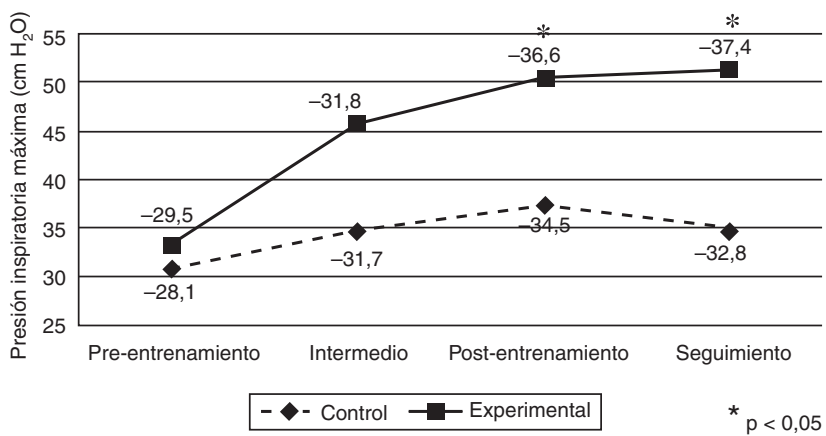

Figura 2. Medias de la presión inspiratoria máxima (PImáx, $\mathrm{cm} \mathrm{H}_{2} \mathrm{O}$ ) para los grupos control y experimental a lo largo de los 4 tiempos: preentrenamiento, intermedio, postentrenamiento y de seguimiento. 


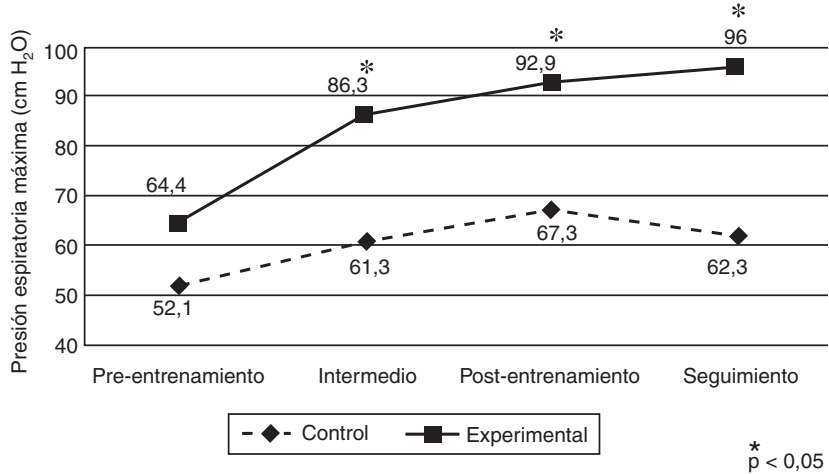

Figura 3. Medias de la presión espiratoria máxima (PEmáx, $\mathrm{cm} \mathrm{H}_{2} \mathrm{O}$ ) para los grupos control y experimental a lo largo de los 4 tiempos: preentrenamiento, intermedio, postentrenamiento y de seguimiento.

efectivo, resultó estadísticamente significativo $\left(F_{3,138}=9,65\right.$; $\left.\mathrm{p}<0,001 ; \eta^{2}=0,173\right)$.

Con relación a la variable relativa a la fuerza de la musculatura espiratoria (PEmáx, $\mathrm{cm} \mathrm{H}_{2} \mathrm{O}$ ), el GE mostró una mejora que se traduce en diferencias significativas entre ambos grupos para las mediciones intermedia (GC=61,3 $\pm 27,4$ [IC95\%; 49,4-73,1] y $\mathrm{GE}=86,3 \pm 30,1$ [IC95\%: 74,4-98,1]; $\mathrm{p}=0,004$ ), postentrenamiento (GC $=67,3 \pm 27,6$ [IC95\%: 55,3-79,3] y GE $=92,9 \pm 30,6$ [IC95\%: $81,0-104,9] ; \quad \mathrm{p}=0,004)$ y de seguimiento (GC=62,3 $\pm 34,9$ [IC95\%: 48,8-75,8] y GE=96,0 $\pm 30,6$ [IC95\%, 82,6-109,5]; $\mathrm{p}=0,001)$. El ANOVA estimado para evaluar el efecto de interacción resultó estadísticamente significativo $\left(F_{3,138}=7,60, \mathrm{p}<0,001\right.$; $\eta^{2}=0,142$ ) (fig. 3).

El comportamiento observado para la fuerza de la MR también se observó para la resistencia de esta (MVV, L/min), que aumentó de forma significativa en el GE para las mediciones postentrenamiento (GC $=33,3 \pm 13,6$ [IC95\%, 27,0-39,6] y GE $=43,1 \pm 16,8$ [IC95\%, 36,8-49,4]; $\mathrm{p}=0,033)$ y de seguimiento (GC $=32,5$ $\pm 16,4$ [IC95\%, 25,8-39,2] y GE=46,1 $\pm 16,2$ [IC95\%, 39,4-52,7]; $\mathrm{p}=0,006)$. En el caso del GE la línea base difiere de todos los demás tiempos $(p>0,05)$, lo que indica estabilidad en el cambio, incluso en el seguimiento. El ANOVA estimado para evaluar el efecto de la interacción resultó estadísticamente significativo $\left(F_{3,138}=9,17\right.$, $\mathrm{p}<0,001 ; \eta^{2}=0,166$ ) (fig. 4).

En cuanto a la satisfacción derivada del entrenamiento, el 92\% de las personas entrenadas contestaron que volvería a participar, mientras que un $4,2 \%$ no supieron qué contestar. Además, apuntaron una media de satisfacción percibida del 8,4 $\pm 2,0$ siendo la puntuación máxima el 10 ("Muy satisfecho»). La media de sesiones de entrenamiento completadas fue de $24 \pm 4$.

Finalmente, se midió el grado de esfuerzo percibido por los mayores durante el protocolo. La puntuación media de la escala

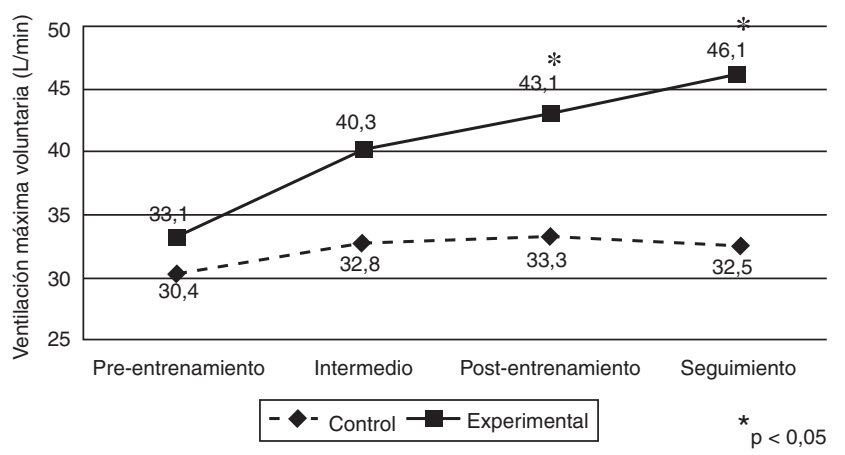

Figura 4. Medias de la ventilación máxima voluntaria (MVV, L/min) para los grupos control y experimental a lo largo de los 4 tiempos: preentrenamiento, intermedio, postentrenamiento y de seguimiento.
Tabla 2

Costes sociales totales del protocolo entrenamiento (2.009€): grupo control (GC, $\mathrm{n}=27)$ y grupo experimental $(\mathrm{GE}, \mathrm{n}=27)$

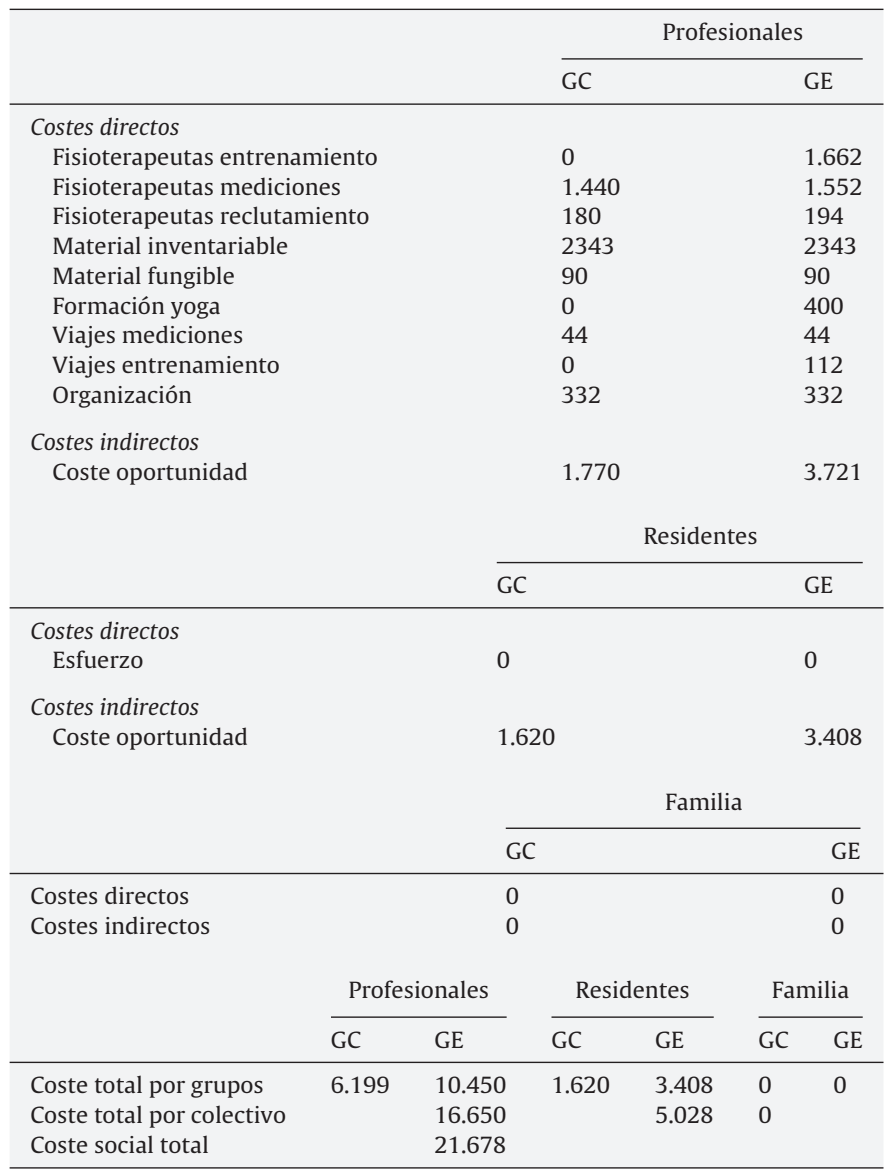

Borg-CR $10^{27}$ durante las 6 semanas de entrenamiento fue de $3,5 \pm 0,6$ no mostrando diferencias estadísticamente significativas $(p>0,05)$ entre las medias semanales, y siendo la puntuación de 3 , un esfuerzo «Moderado».

\section{Costes sociales de la intervención}

Tras la estimación correspondiente según los parámetros indicados en la metodología, el total de costes directos e indirectos de los profesionales ascendió a $16.650 €$ (tabla 2). En lo que respecta a los residentes, consideramos unos costes directos nulos debido a que solo mostraron un grado moderado de esfuerzo (según BorgCR10) propio de la adaptación al entrenamiento respiratorio y necesario para la obtención de la mejora respiratoria. Sin embargo, sus costes indirectos (la actividad que dejaron de realizar en la residencia, y que normalmente consistía en otras actividades lúdicas programadas por la residencia) ascendieron a 5.028€. Por último, los costes directos e indirectos de la familia fueron nulos, dado que percibieron que sus mayores estaban más activos y esto no alteró su comportamiento habitual de visitas, ni supuso reclamaciones o quejas. En resumen, los costes sociales totales (directos e indirectos, para el GC y GE) de esta intervención fueron de $21.678 €$.

\section{Discusión}

Este es el primer ensayo controlado aleatorizado en el que se describen y estiman los costes sociales y resultados de un programa de prevención respiratoria en el anciano institucionalizado con limitación funcional para las AVD. En respuesta al objetivo 
planteado en este estudio, podemos señalar que a través de un programa de Pranayama se puede mejorar la función de la MR, tanto en lo que respecta a la fuerza (PImáx y PEmáx) como a la resistencia (MVV) de la MR.

Con relación a estudios previos, nuestros resultados se suman a la evidencia de los efectos positivos del Pranayama a nivel de la función respiratoria ${ }^{12-14}$, pero en este caso en una población de edad avanzada (edad media $85 \pm 6$ años), con comorbilidad y especialmente debilitada. En la línea de nuestros resultados, Santaella et al. ${ }^{14}$ observaron un mayor incremento en la PEmáx (34\%, $\mathrm{p}<0,001)$ que en la PImáx $(26 \%, \mathrm{p}<0,001)$, pero en este caso la población anciana vivía en comunidad y realizaba desplazamientos regularmente (edad media $68 \pm 6$ años). En este mismo estudio ${ }^{14}$ se argumenta que la respiración lenta en la que se intercalan la contracción isotónica concéntrica de la musculatura inspiratoria, la contracción isométrica postinspiratoria y la contracción isotónica e isométrica de la MR, parecen mejorar la capacidad de la caja torácica para generar presiones negativas y positivas durante el proceso de la respiración. Por otra parte, el diseño de investigación desarrollado en este estudio permite comparar la evolución de las variables resultado a través de mediciones consecutivas y de seguimiento, a diferencia de los estudios previos en los que se realizan la medición pre y postintervención ${ }^{12-14}$.

Con relación al grado de satisfacción percibida por el GE, la valoración positiva indica que la calidad de este protocolo según sus participantes es alta. Este es un hecho a destacar, dado el tipo de colectivo y contexto tan especial al que se aplica. A este respecto, Santaella et al. ${ }^{14}$ destacan que su muestra estaba compuesta por ancianos voluntarios sanos y altamente motivados, y que por lo tanto, los resultados que obtuvieron no se podían extrapolar a la población anciana con comorbilidad. Si a esto le añadimos las observaciones de Simões et al. $^{4}$ respecto al anciano institucionalizado, podemos afirmar que la intervención mediante Pranayama se pudo acompañar de cierto grado de motivación al observar el elevado cumplimiento ( $24 \pm 4$ sesiones completadas).

Respecto a los costes sociales directos e indirectos analizados, comprobamos que en general son moderados y recaen básicamente sobre los profesionales. Una vez descritos y comparados resultados con costes de la intervención, se percibe un predominio de efectos positivos frente a los negativos. Con esta sencilla evaluación hemos contribuido a generar información relevante para los gestores interesados en intervenciones centradas en la persona ${ }^{15}$ en el ámbito de las residencias y de rehabilitación, y se comprueba que es una intervención fácilmente asumible por las residencias. Esto está en consonancia con lo que indica la revisión de intervenciones centradas en la persona y preventivas realizada por la Fundación Matia ${ }^{17}$, que en su mayoría se muestran costeefectivas.

Como limitaciones de esta intervención y futuras mejoras de este estudio, se deberían incluir: a) un grupo placebo, a fin de poder medir los efectos del entrenamiento y evitar la posible influencia del aprendizaje, al que atribuimos la tendencia a la mejora observada en el GC; b) otros resultados como la mejora en la disnea y la calidad de vida. Al respecto de la calidad de vida asociada a parámetros respiratorios y para la población anciana institucionalizada con amplia comorbilidad e incapacidad para deambular, debemos apuntar que no existen cuestionarios validados, de ahí la necesidad de su elaboración y validación previas. Por otra parte, se podría comparar el Pranayama con otras intervenciones de fisioterapia respiratoria y estimar un coste-efectividad.

Como conclusión, esta evaluación revela que los costes sociales del protocolo son moderados, aun cuando los fisioterapeutas son externos a la residencia, que los resultados en términos de la función de la MR son significativos, y que la intervención es bien tolerada y valorada por el residente. Por otra parte, las características de la población hacen especialmente relevantes estas conclusiones, tanto por su edad como por sus condiciones de funcionalidad general y específica de la MR.

Clínicamente, mejorar la función de la MR supone una mayor reserva funcional respiratoria, que ante la presencia de complicaciones y/o exacerbaciones frecuentes en esta población puede evitar casos de insuficiencia respiratoria que ocasionan el ingreso hospitalario y/o la mortalidad.

\section{Conflicto de intereses}

Los autores declaran no tener ningún conflicto de intereses.

\section{Agradecimientos}

Los autores quieren agradecer a todos los mayores residentes su participación, y a la dirección y personal sociosanitario de las residencias de LARES Comunidad Valenciana que han permitido y colaborado en el desarrollo de este estudio. También queremos mostrar nuestro agradecimiento a todos los fisioterapeutas que han participado en el estudio. Laura Galiana es becaria del Programa VLC/Campus, subprograma Atracció de Talent.

\section{Bibliografía}

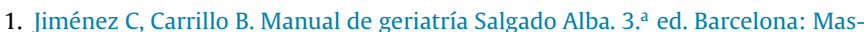
son; 2002.

2. Summerhill EM, Angov N, Garber C, McCool FD. Respiratory muscle strength in the physically active elderly. Lung. 2007;185:315-20.

3. Watsford ML, Murphy AJ, Pine MJ. The effects of ageing on respiratory muscle function and performance in older adults. J Sci Med Sport. 2007;10:36-44.

4. Simões RP, Castello V, Auad MA, Dionísio J, Mazzonetto M. Prevalence of reduced respiratory muscle strength in institutionalized elderly people. Sao Paulo Med J 2009;127:78-83

5. Belman MJ, Gaesser GA. Ventilatory muscle training in the elderly. J Appl Physiol. 1988;64:899-905.

6. De Andrade AD, Silva TN, Vasconcelos H, Marcelino M, Rodrigues-Machado MG, Filho VC, et al. Inspiratory muscular activation during threshold therapy in elderly healthy and patients with COPD. J Electromyogr Kinesiol. 2005;15:631-9.

7. Watsford ML, Murphy AJ. The effects of respiratory-muscle training on exercise in older women. J Aging Phys Activ. 2008;16:245-60.

8. Cebrià i Iranzo MD, Arnall DA, Igual Camacho C, Tomás JM, Meléndez JC. Physiotherapy intervention for preventing the respiratory muscle deterioration in institutionalized older women with functional impairment. Arch Bronconeumol. 2013;49:1-9.

9. Hill K, Cecins NM, Eastwood PR, Jenkins SC. Inspiratory muscle training for patients with chronic obstructive pulmonary disease: A practical guide for clinicians. Arch Phys Med Rehabil. 2010;91:1466-70.

10. Gosselink R, de Vos J, van den Heuvel SP, Segers J, Decramer M, Kwakkel G. Impact of inspiratory muscle training in patients with COPD: What is the evidence? Eur Respir J. 2011;37:416-25.

11. Danucalov MA, Simões RS, Kozasa EH, Leite JR. Cardiorespiratory and metabolic changes during yoga sessions: The effects of respiratory exercises and meditation practices. Appl Psychophysiol Biofeedback. 2008;33:77-81.

12. Madanmohan Udupa K, Bhavanani AB, Vijayalakshmi P, Surendiran A. Effect of slow and fast pranayams on reaction time in cardiorespiratory variables. Indian J Physiol Pharmacol. 2005;49:313-8.

13. Madanmohan SK, Mahadevan SB, Balakrishnan S, Gopalakrishnan M, Prakash ES. Effect of six weeks yoga training on weight loss following step test, respiratory pressures, handgrip strength and handgrip endurance in young health subjects. Indian J Physiol Pharmacol. 2008;52:164-70.

14. Santaella DF, Devesa CR, Rojo MR, Amato MB, Drager LF, Casali KR, et al Yoga respiratory training improves respiratory function and cardiac sympathovagal balance in elderly subjects: A randomised controlled trial. BMJ Open. 2011;24:e000085.

15. Martínez Rodríguez T. La atención gerontológica centrada en la persona. Gizartea Hobetuz. Documentos de bienestar social. Vitoria-Gasteiz: Servicio central de publicaciones del Gobierno Vasco; 2011

16. Asociación de Economía de la Salud. La sanidad pública ante la crisis. Recomendaciones para una actuación pública sensata y responsable. Documento de debate (acceso 16 Abr 2013). Disponible en: http://aes.es/Publicaciones/ DOCUMENTO_DEBATE_SNS_AES.pdf

17. Fundación Matia Atención comunitaria y atención centrada en la persona: revisión de estudios de coste-efectividad. Vitoria-Gasteiz: Centro de Documentación y Estudios SIIS; 2011

18. OECD. A good life in old age? Monitoring and improving quality in long-term. Brussels: OECD; 2013 (acceso 18 Jun 2013). Disponible en: 
http:/www.keepeek.com/Digital-Asset-Management/oecd/social-issuesmigration-health/a-good-life-in-old-age_9789264194564-en

19. Drummond MF, O'Brien BJ, Stoddart GL, Torrance GW. Métodos para la evaluación económica de los programas de asistencia sanitaria. 2. ${ }^{a}$ ed. Madrid: Díaz de Santos; 2001

20. Coast J. Is economic evaluation in touch with society's health values? BMJ 2004:329:1233-6.

21. Trueman P, Anokyen NK. Applying economic evaluation to public health interventions: The case of interventions to promote physical activity. J Public Health (Oxf). 2012;35:32-9.

22. Gage $\mathrm{H}$, Kaye J, Owen C, Trend P, Wade D. Evaluating rehabilitation using cost-consequences analysis: An example in Parkinsons's disease. Clin Rehabil. 2006;20:232-8.

23. Artaso Irigoyen B, Martín Carrasco M, Cabasés Hita JM. Análisis costeconsecuencia de un centro de día psicogeriátrico. Rev Esp Geriatr Gerontol. 2002;37:291-7.

24. James M, Stokes EA, Thomas E, Dziedzic K, Hay EM. A cost consequences analysis of local corticosteroid injection and physiotherapy for the treatment of new episodes of unilateral shoulder pain in primary care. Rheumatology (Oxford) 2005; $44: 1447-51$.

25. Tortosa MA, Fuenmayor A, Granell R. Evaluación de costes y financiación de las residencias de mayores. El sector no lucrativo en la Comunidad Valenciana. Madrid: Informes Portal Mayores, $\mathrm{n}^{\circ}$ 129; 2011 (acces 20 Dic 2011). Disponible en: http://www.imsersomayores.csic.es/documentos/ documentos/tortosa-evaluacion-01.pdf

26. Lobo A, Saz P, Marcos G. Adaptación del Examen Cognoscitivo Mini-Mental. Madrid: Tea Ediciones; 2002.

27. Borg G. The Borg CR10 scale folder. A method for measuring intensity of experience. Hasselby, Sweden: Borg Perception; 2004.
28. Enright PL, Kronmal RA, Manolio TA, Schenker MB, Hyatt RE. Respiratory muscle strength in the elderly: Correlates and reference values. Am J Respir Crit Care Med. 1994;149 2 Pt 1:430-8.

29. Neder JA, Andreoni S, Lerario MC, Nery LE. Reference values for lung function tests. II. Maximal respiratory pressures and voluntary ventilation. Braz J Med Biol Res. 1999;32:719-27.

30. Miller MR, Hankinson J, Brusasco V, Burgos F, Casaburi R, Coates A, et al. Standard of spirometry. Eur Respir J. 2005;26:319-38.

31. American Thoracic Society/European Respiratory Society. ATS/ERS. Statement on respiratory muscle testing. Am J Respir Crit Care Med. 2002;166:518624.

32. Diari Oficial de la Comunitat Valenciana. Resolución de 26 de marzo de 2009 de la Dirección General de Trabajo, Cooperativismo y Economía Social, por la que se dispone el registro y publicación del acuerdo de la Comisión Paritaria de Convenio Colectivo para el Sector Privado de Residencias para la Tercera Edad, Servicios de Atención a las Personas Dependientes y Desarrollo de la Promoción de la Autonomía Personal en la Comunidad Valenciana sobre revisión salarial para el año 2009. DOCV núm. 6006 de 5/5/2009.

33. Diari Oficial de la Comunitat Valenciana. Orden de 17 de diciembre de 2008 de la Conselleria de Infraestructuras y Transporte por la que se actualizan las tarifas de los servicios de transporte público de viajeros de Ferrocarriles de la Generalitat Valenciana así como las de los títulos de uso en el área de transporte metropolitano de Valencia. DOCV núm. 5919 de 17/12/2008.

34. Universitat de València (Estudi General). Pressupost i reglament d'execució per a 2009.

35. Boletín Oficial de la Provincia de Valencia. Anuncio de la Conselleria de Economía, Hacienda y Empleo sobre texto del convenio colectivo de trabajo del sector de sanidad privada de la provincia de Valencia. BOP núm. 298 de $10 / 12 / 2008$ 